\title{
Manifestaciones orales del síndrome de Mobius asociado a Poland: descripción de un caso clínico
}

\section{Oral manifestations of Mobius syndrome associated to the Poland anomaly: a case report}

\author{
Figueiredo MC*, Faustino-Silva DD**, Bez AS***, Rincón DPC****
}

\section{RESUMEN}

El síndrome de Mobius es una anomalía congénita que causa parálisis de los nervios VI y VII pares craneanos. Se caracteriza por malformaciones límbicas y cráneo-buco-faciales. Asociada a Poland, presenta malformación del músculo pectoral mayor y anomalías en pies y manos. Por ser un síndrome que presenta anomalías faciales y orales importantes como paladar ojival, mordida abierta, xerostomía, úvula bífida, malformaciones en la lengua, se destaca la necesidad de un abordaje odontológico eficaz con el fin de establecer una adecuada salud oral y calidad de vida del paciente. En este trabajo, descripción de un caso clínico, se expone los aspectos clínicos generales y orales, así como el tratamiento odontológico realizado en una paciente portadora del síndrome Mobius/Poland. Un abordaje enfocado en la orientación del responsable y en el control de la caries y la enfermedad periodontal, encontró una reducción significativa en los índices de placa bacteriana y sangrado gingival. Actualmente la paciente se encuentra en fase de mantenimiento. Como no hay cura para el síndrome, el tratamiento es enfocado al control y mantenimiento de las manifestaciones orales.

Palabras clave: Síndrome de Poland, síndrome de Mobius, manifestaciones orales, salud oral.

\section{SUMMARY}

The Mobius syndrome is a congenital paralysis of the sixth and seventh cranial nerves. It is characterized by craniofacial and limb malformations. When it is associated to the Poland, the Mobius syndrome shows aplasia of the pectoral muscle (Poland anomaly) and abnormalities of the feet and hands. The syndrome shows important oral and facial abnormalities like gothic palate, open bite, dry mouth, bifid uvula, tongue alterations, and because of this, there is the necessity of an effective odontological approach in order to establish an adequate oral health and patient's quality of life. In this study, the clinical case report shows the oral and general clinical aspects, as well as the odontological treatment made in a patient who has the Mobius/Poland syndrome. After the approach focus on the orientations to the carer and in the control of the periodontal and carie diseases, it was realized a significative reduction in the visible plaque index (VPI) and gum bleeding index

* Profesora de Odontopediatría. Facultad de Odontología. Universidad Federal de Río Grande do Sul. Brasil.

** Especialista en Salud Colectiva, y Odontopediatría. Facultad de Odontología. Universidad Federal de Río Grande do Sul. Brasil.

*** Odontóloga. Alumna interna del Curso de Extensión Universitaria Atención de Pacientes Especiales de la Facultad de Odontología. Universidad Federal de Río Grande do Sul. Brasil.

**** Odontóloga. Exalumna del Curso de Extensión Universitaria Facultad de Odontología. Universidad Federal de Río Grande do Sul. Brasil. 
(GBI). Currently, the patient is in maintenance. The syndrome is incurable; therefore, the treatment is based on the control and maintenance of oral manifestations.

Key words: Poland syndrome, Mobius Syndrome, Ooral manifestations, oral health.

Fecha de recepción: 19 de junio 2008.

Aceptado para publicación: 30 de junio 2008.

Figueiredo MC, Faustino-Silva DD, Bez AS, Rincón DPC. Manifestaciones orales del síndrome de Mobius asociado al síndrome de Poland: descripción de un caso clínico. Av. Odontoestomatol 2009; 25 (4): 179-183.

\section{INTRODUCCIÓN}

Se describe la asociación de las anomalías Mobius y Poland manifestación rara en la literatura. Mientras que algunos autores creen que es una asociación independiente, otros afirman que Poland, Mobius y Poland/Mobius son variaciones de la misma condición (1).

El síndrome de Mobius es una alteración congénita rara que se caracteriza por la parálisis de los nervios VI y VII pares craneanos, que puede ser completa o parcial, unilateral o bilateral, que resulta en un rostro inexpresivo o poco expresivo (2). Otros signos son las malformaciones límbicas (estructuras responsables por las emociones y comportamientos) y bucofacial.

La etiología y patogenia del síndrome de Mobius es dudosa, pero existen dos teorías: genética (gen autosómico dominante) e isquémica, considerando la teratogenicidad como un factor etiológico relevante para ambos (3).

Las malformaciones cráneo-buco-faciales pueden comprender asimetría facial, patosis palpebral, estrabismo convergente, hipertelorismo, sordera, malformación del oído externo, base de la nariz ancha, falta del párpado inferior, pliegues excéntricos, disfagia, dificultad para succionar, microstomía, micrognasia, asimetría de la comisura labial, alteraciones de la lengua (hipoglosia, aglosia o anquiloglosia), fisura palatina, úvula bífida, paladar alto y oligodoncia $(3,4)$. La malformación (aplasia) del músculo pectoral mayor y simbraquidactilia conforman la asociación del síndrome de Poland al Mobius (1).

El síndrome de Mobius, por ser una anomalía craneofacial, con frecuencia causa problemas periodontales, lesiones cariosas, deformidades en la lengua y paladar (5). Aquí el papel fundamental del odontólogo en el manejo de estos pacientes, proporcionando salud oral que influye en una mejor calidad de vida. En este sentido, se destaca la importancia de la capacitación de los profesionales en el tratamiento de pacientes portadores de necesidades especiales.

El objetivo de este articulo es relatar el acompañamiento clínico odontológico de una paciente portadora del síndrome de Mobius asociado a Poland, desde su infancia hasta la vida adulta, con énfasis en el abordaje de la educación de sus familiares responsables.

\section{DESCRIPCIÓN DEL CASO CLÍNICO}

La paciente, de sexo femenino, de 26 años de edad, tez blanca, portadora de síndrome de Mobius/Poland, inició el tratamiento odontológico a los 11 meses de edad, en la clínica de Odontopediatría de la Facultad de Odontología de la Universidad Federal de Río Grande do Sul/UFRGS-Brasil, donde recibió un tratamiento educativo-preventivo. Pasados 23 años, regresa a la Facultad, allí fue atendida en la Clínica de Atención Odontológica a Pacientes Portadores de Necesidades Especiales, donde el padre refiere que la paciente presenta dolor de diente. 
El diagnóstico del síndrome de Mobius asociado a Poland fue realizado cuando la paciente tenía 8 años de edad, por el equipo de Genética Médica del Hospital de las Clínicas de Porto Alegre.

En la anamnesis, la madre niega el uso de drogas perjudiciales al feto durante la gestación; padres saludables y sin consanguinidad y nacimiento por parto normal.

En el examen clínico general, se encuentra: agenesia del músculo pectoral derecho (asociado a la anomalía de Poland), estrabismo convergente, obesidad mórbida (causada por la ingesta de psicotrópicos indicados en el tratamiento para evitar la automutilación), rostro inexpresivo (Fig. 1), sindactilia (dedos fusionados) en las manos derecha (Fig. 2) e izquierda, retraso mental profundo, alto grado de autismo, audición disminuida e incapacidad de habla.

Al examen extraoral, se observa hipoplasia del labio superior, queilitis angular y respiración oral. Ya, en el examen intraoral, hay presencia de mordida abierta anterior, macroglosia (Fig. 3), paladar alto, sangrado gingival generalizado, índice de placa bacteriana visible igual al $100 \%$, cavidad profunda cariosa y activa en el 18 y erupción ectópica palatina de 15, que dificulta la masticación.

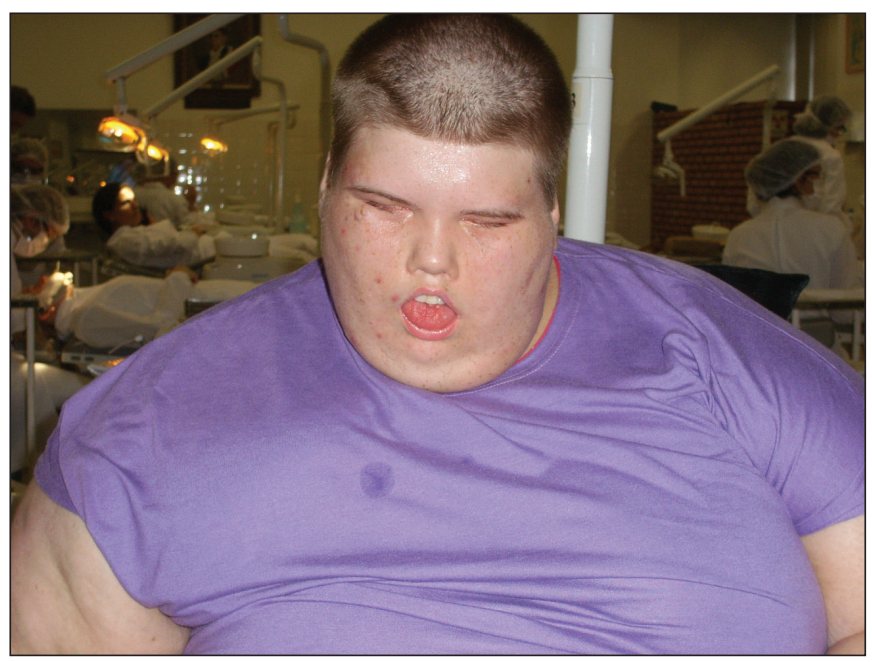

Fig. 1. Aspecto físico general de la paciente, donde se evidencia la agenesia del músculo pectoral derecho, estrabismo convergente, obesidad mórbida, rostro inexpresivo, macroglosia e hipotonía lingual.

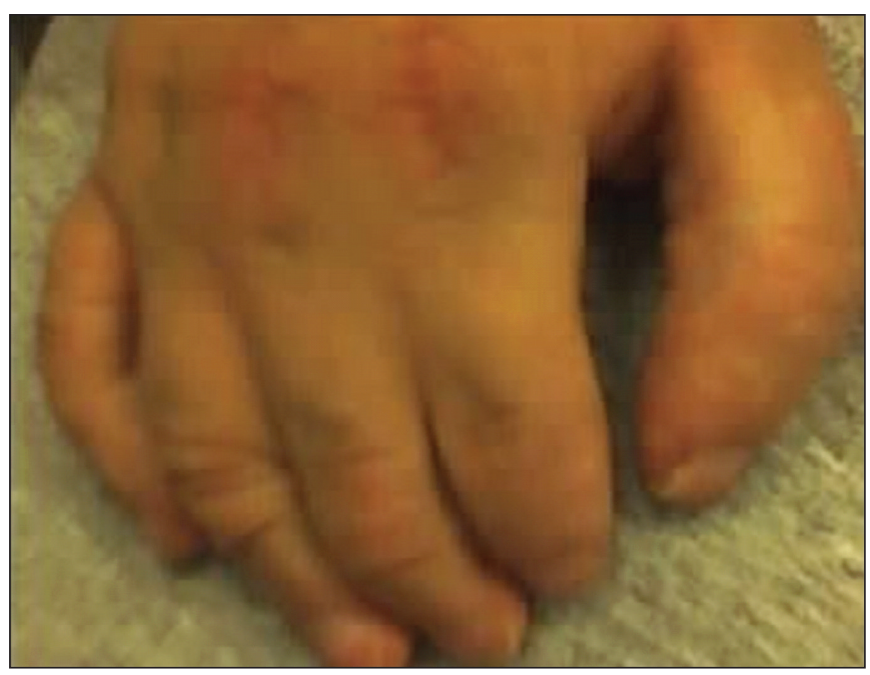

Fig. 2. Presencia de sindactilia (dedos fusionados) en la mano derecha.

El tratamiento odontológico hace énfasis en la instrucción de higiene oral al familiar responsable por la paciente y aplicación tópica de flúor durante cuatro semanas, acompañado por raspado gingival de todos los sectantes y profilaxis.

Se realizaron las exodoncias de los dientes: 15, por su erupción ectópica por palatino, y del 18 debido a la lesión cariosa activa profunda.

Simultáneamente, se prescribió control químico de la placa bacteriana con clorhexidina al $2 \%$ en gel,

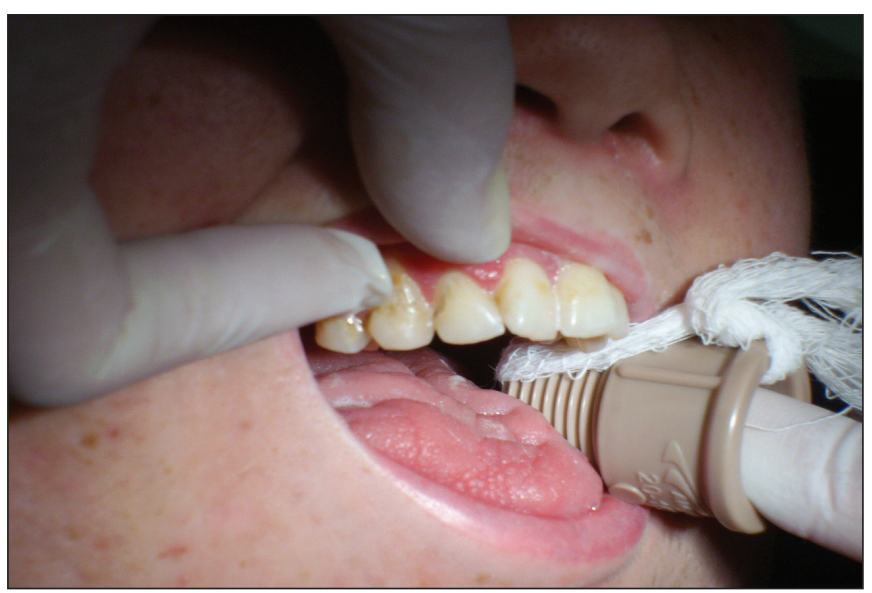

Fig. 3. Aspecto oral de la paciente después del tratamiento y el uso del mantenedor de apertura alternativo. 
por medio de dos aplicaciones diarias y cepillado correcto.

Después del tratamiento, se obtiene una mejoría en el cuadro clínico odontológico de la paciente: disminución de los índices de placa visible y sangrado gingival (ISG $=30 \%$ ), ausencia de lesiones cariosas activas y cálculo dental (Fig. 3).

La paciente se encuentra en fase de mantenimiento, realizada cada 3 meses en la Facultad de Odontología de la Universidad Federal de Río Grande do Sul, con el acompañamiento del equipo de Genética Médica del Hospital de las Clínicas de Porto Alegre.

\section{DISCUSIÓN}

La presentación del síndrome de Mobius/Poland es rara. Segun Strömland et al. 2002 (6), de los 25 pacientes con síndrome de Moebius, solamente dos presentaban Poland. Silvani et al. 1981 (7), asocia Poland a Mobius en $13 \%$ de los casos.

El tratamiento odontológico de los pacientes con síndrome de Mobius es limitado por la movilidad y desarrollo de la musculatura de la cavidad oral y perioral disminuido, dificultando así, la práctica de una higiene oral adecuada (8). Para alcanzar un acondicionamiento bucal del paciente, se hace necesaria una acción educativa con las personas responsables del paciente, paralela a la intervención profesional.

Después de 23 años, la paciente regresó a la facultad, pues presentó sintomatología dolorosa en el diente 18. El tratamiento indicado fue la exodoncia, porque presentaba una cavidad profunda activa, con compromiso pulpar e indicación de endodoncia. Sin embargo es conocido que el tratamiento de conductos de un tercer molar presenta limitaciones técnicas agravadas por las características sindrómicas de la paciente, razón por la cual se toma la decisión de realizar la exodoncia. Por las mismas dificultades asociadas al síndrome, impiden que se lleve a cabo el mantenimiento de un tratamiento ortodóncico, se realizó la exodoncia del 15 que estaba posicionado fuera del arco dental, por palatino. Los procedimientos invasivos fueron realizados en dos sesiones. Las personas portadoras de este síndrome son más vulnerables a sufrir enfermedades bucales debido a la disminución de la función de los músculos masticatorios, lengua hipoplásica, y ausencia de sellado perioral (9), dificultando así la higiene oral. Se puede decir, que las lesiones cariosas pueden ser atribuidas, en parte, a la higiene oral deficiente y a una dieta inadecuada, que normalmente es pastosa y azucarada. Niños o adultos con dificultad de aprendizaje y anomalías límbicas son incapaces de cooperar con un cuidado diario de higiene oral, aumentando de esta forma, la incidencia de caries y enfermedad periodontal (8). Conforme con este mismo estudio, la enfermedad periodontal se observó en todos los cuadrantes en la mayoría de los doce pacientes analizados. Entendemos, entonces, la importancia de realizar un abordaje educativo al adulto responsable por el paciente, explicándole las causas de ambas enfermedades para que el cuadro clínico se mantenga estable, es decir, sin la presencia de enfermedades activas.

El tratamiento se da por la intervención adecuada del profesional conjuntamente con el cuidado en casa, incentivando la higiene oral diaria con métodos apropiados, dando como resultado una salud oral que contribuya con una mejora del estado general del paciente. Un método que facilita el examen oral y los procedimientos odontológicos es el uso de un mantenedor de apertura, ya que muchos pacientes con necesidades especiales presentan sistema nervioso central comprometido, movimientos involuntarios o hipotónicos, además de macroglosia. El mantenedor de apertura alternativo (Fig. 3) consiste en un trozo de tubo de PVC que es de bajo costo y se encuentra fácilmente en almacenes de materiales de construcción, además de adaptarse bien al dedo índice del profesional, protege de posibles mordidas al responsable permitiendo una adecuada higienización diaria de la cavidad oral de forma práctica y segura, facilitando el mantenimiento de la salud oral del paciente especial.

El abordaje preventivo fue el eje del tratamiento, por medio de raspados supragingivales y pulido dentario, aplicaciones tópicas de flúor para controlar la actividad cariosa de la paciente. Además, la orientación para la higienización diaria con clorhexidina $2 \%$ en gel se justifica por la dificultad del control mecánico aislado, destacando la utilización de ambas técnicas. La educación se efectuó por medio de la ex- 
Figueiredo MC, Faustino-Silva DD, Bez AS, Rincón DPC. Manifestaciones orales del síndrome de Mobius asociado al síndrome de Poland: descripción de un caso clínico

plicación de los hábitos de higiene oral y orientación de la dieta al responsable del paciente. Las consultas ambulatorias realizadas en la Facultad de Odontología de la Universidad Federal de Río Grande do Sul fueron posibles gracias a la ayuda del padre y al manejo de comportamiento realizado por los profesionales que la atendieron. El manejo ambulatorial, en detrimento del hospitalario, se vuelve menos traumático y con menos riesgos para el paciente, así como su menor costo. La paciente sera acompañada en consultas de mantenimiento, ya que el tratamiento propuesto fue concluido. Es importante resaltar que la paciente siempre tuvo acompañamiento médico, manteniendo una interacción entre los profesionales que la trataron. La paciente hace visitas periódicas al médico y al odontólogo, tratando los síntomas del síndrome de manera integral, con el fin de alcanzar mejoras en su calidad de vida.

Por presentar un cuadro de parálisis facial, la higiene oral es difícil y el riesgo de enfermedades orales son mayores y precoces en pacientes con síndrome de Mobius/Poland. La intervención odontológica debe realizarse temprano, debido a las características inherentes a este síndrome. El abordaje multidisciplinario es esencial para un tratamiento adecuado, además, el compromiso con la familia, el desarrollo de una relación de confianza con los profesionales deben ser la pauta de toda terapéutica de tratamiento del paciente con síndrome de Mobius/Poland.

\section{BIBLIOGRAFÍA}

1. Domingos AC, Lopes SLCP, Almeida SSM, Boscolo FN, Whaites EJ. Poland-Moebius syndrome: a case with oral anomalies.Oral Diseases 2004;10(6):404-7.

2. Scalisi F.C. Espectro clínico y etiológico del síndrome de Moebius. Arch. Argent. Pediatric 2007; 105(5):444-6.
3. Magalhães MHCG, Araújo LCA, Chiaradia CC, Fraigea A, Zamunaro MT, Mantesso A. Early dental management of patients qith Mobius syndrome. Oral Diseases 2006;12(6):533-6.

4. Freitas AC, de Filho PN, Queiroz AM, de Assed S, Silva FWGP. Síndrome de Moebius: relato de caso clínico. Revista de Odontologia da Universidade Cidade de São Paulo 2006;18(3):297-302.

5. Aren D. Mobius syndrome: a case report. The Journal Clinical of Pediatric Dentistry 2002;26 (2):207-9.

6. Strömland K, Sjögreen L, Miller M, Gillberg C, Wentz E. Johansson M. Nylén O. et al. Möbius sequence-a Swedish multidiscipline study. European Journal of Pediatric Neurology 2002; 6(1):35-45.

7. Silvani SH, de Valentine SJ, Karlin JM, Scurran BL. Möbius'syndrome and talipes equinovarus. Journal of American Pediatric Association 1981;74(11):604-6.

8. De Serpa-Pinto MVX, de Magtalhães MHCG, Nunes FD, Nunes FD. Moebius syndrome with oral envolvement. International Journal of Pediatric Dentistry 2002;12(6):446-9.

9. Ling KJ, Wang WN. Moebius syndrome-report of case. Journal of Dentistry Child 1997;64(1): 64-7.

\section{CORRESPONDENCIA}

Rua Luzitana 1370/502

Higienópolis - Porto Alegre-RS, Brasil

CEP: 90520-080

Teléfono: (51) 3308-5026

E-mail:mcf1958@gmail.com 